Original Paper http://ajol.info/index.php/ijbcs http://indexmedicus.afro.who.int

\title{
Impact des aliments thérapeutiques sur les paramètres biochimiques des enfants malnutris en Guinée
}

\author{
Falaye TRAORE ${ }^{1,2}$, Mamadou Saliou BAH ${ }^{3,4}$, Sidikiba SIDIBE $^{5 *}$, Benjamin LAMAH ${ }^{1}$, \\ Amara Tabaouo SAMAKE ${ }^{5}$ et Ahmadou Sadio DIALLO ${ }^{6}$ \\ ${ }^{1}$ Laboratoire des Sciences Physicochimiques Pharmaceutiques, Faculté de Médecine-Pharmacie- \\ Odontostomatologie, Université Gamal Abdel Nasser de Conakry, B.P. 1147, Conakry, Guinée. \\ ${ }^{2}$ Institut National de Santé Publique, B.P. 6623, Conakry, Guinée. \\ ${ }^{3}$ Laboratoire de Biochimie, Nutrition, Toxicologie, Faculté de Médecine-Pharmacie-Odontostomatologie, \\ Université Gamal Abdel Nasser de Conakry, B.P. 1147, Conakry, Guinée. \\ ${ }^{4}$ Laboratoire Médical Guinéo Allemand, Corniche Nord, Commune de Dixinn, B.P. 4925, Conakry, Guinée. \\ ${ }^{5}$ Chaire Santé Publique, Faculté de Médecine Pharmacie Odonto stomatologie, \\ Université Gamal Abdel Nasser, Conakry, Guinée (UGANC) \\ ${ }^{6}$ Laboratoire de Biochimie, Faculté des Sciences, \\ Université Gamal Abdel Nasser de Conakry, B.P. 1147, Conakry, Guinée. \\ "Auteur correspondant; E-mail: layesidikiba@gmail.com; Tel: 00224628993899
}

\section{RESUME}

L'utilisation des aliments thérapeutiques prêts à l'emploi (ATPE) dans la prise en charge des malnutritions est devenue fréquente en raison de la forte prévalence des maladies carentielles chez les enfants. L'objectif de cette étude était d'évaluer leur impact sur les paramètres biochimiques et anthropométriques des enfants malnutris à l'Institut de Nutrition et de Santé de l'Enfant (INSE) de Guinée. Il s'agissait d'une étude prospective portant sur 41 enfants atteints de malnutrition aigüe sévère soumis à une récupération nutritionnelle pendant 6 mois au cours desquels leurs paramètres staturo-pondéraux et biochimiques ont été appréciés. Nous avons observé dans l'ensemble une normalisation des paramètres staturo-pondéraux, avec des poids variant entre 7,0 à 9,6 kg, correspondants à des gains poids de 800 à $1600 \mathrm{~g}$ respectivement. Quant aux paramètres biochimiques, ils se sont normalisés dans $60 \%$ des cas au cours d'une durée moyenne d'hospitalisation de 24 jours. Les aliments thérapeutiques ont montré une influence positive sur les paramètres staturo-pondéraux et biochimiques des enfants malnutris. Ils devraient donc être utilisés pour lutter contre la malnutrition chez eux en veillant toutefois à contrôler leurs paramètres ioniques dont les perturbations peuvent nuire à leur santé.

(c) 2018 International Formulae Group. All rights reserved.

Mots clés : Enfants, malnutrition, aliments thérapeutiques, récupération nutritionnelle.

\section{Impact of therapeutic foods on biochemical parameters of malnourished children in Guinea}




\begin{abstract}
The use of ready-to-use therapeutic foods (RUTF) in the management of severe acute malnutrition is increasingly common due to the high prevalence of childhood illnesses. The objective of this study was to evaluate their impact on the biochemical and anthropometric parameters of severely malnourished children at the Institute of Children Nutrition and Health in Guinea. This was a prospective study on 41 children with severe acute malnutrition who underwent nutritional recovery for 6 months during which their anthropometric and biochemical parameters were assessed. On the whole, we observed a normalization of the anthropometric parameters, with weights ranging from 7.0 to $9.6 \mathrm{~kg}$, corresponding to weight gains of 800 to $1600 \mathrm{~g}$ ( $90 \%$ and $102 \%$ of the target weights, respectively). As for the biochemical parameters, they were standardized in $60 \%$ of the cases over an average hospital stay of 24 days. Therapeutic foods have shown a very positive influence on the biochemical and statistical parameters of malnourished children. They should therefore be used to combat malnutrition in their homes, while ensuring that their ionic parameters are controlled and that their disturbances can be harmful to their health.
\end{abstract}

(C) 2018 International Formulae Group. All rights reserved.

Keywords: Children, malnutrition, therapeutic feeding, nutritional recovery,

\section{INTRODUCTION}

L'OMS définit la malnutrition aiguë sévère (MAS) chez les enfants de 6 à 59 mois comme un rapport poids/taille inférieur de -3 écarts types (z-scores) aux normes de croissance de l'OMS ou un périmètre brachial inférieur à $115 \mathrm{~mm}$, avec ou sans œdèmes bilatérale des membres inférieurs (Ashworth et al., 2003 ; OMS et UNICEF, 2009 ; WHO, 2013).

La malnutrition aiguë sévère touche environ 20 millions d'enfants de moins de cinq ans et est associée à 1-2 millions de décès qui pourraient être évités chaque année. En Afrique, plus de $30 \%$ des décès chez les enfants de moins de 5 ans sont imputables à la malnutrition (Fergusson and Tomkins, 2009).

En 2005 en Guinée, 9,4\% des enfants de 6 à 59 mois étaient malnutris dans les quatre régions administratives du pays (avec une prévalence supérieure à $10 \%$ considérée comme le seuil d'alerte). Cela s'expliquerait par des pratiques inadéquates d'alimentation du petit enfant. En effet, seulement $27 \%$ des enfants de moins de 6 mois sont allaités exclusivement au lait maternel, tandis que $35 \%$ des décès d'enfants guinéens de moins d'un an sont attribuables à des pratiques inadéquates d'allaitement maternel. Par contre, $45 \%$ des enfants de 6 à 9 mois ainsi qu'un nombre élevé d'enfants de moins de 5 ans malnutris ne recevaient pas encore d'aliments de complément indispensables à leur croissance (MSHP, 2008).
L'appréciation de l'état nutritionnel d'un membre d'une collectivité est basée sur un certain nombre d'examens cliniques, biochimiques, anthropométriques et biophysiques. L'avantage de l'évaluation biochimique de la nutrition consiste en la possibilité de détecter des changements, avant d'arriver à un stade où les perturbations biologiques et cliniques deviennent apparentes. En effet, les valeurs biochimiques renseignent sur la concentration tissulaire des éléments nutritifs ainsi que sur toute présence d'anomalie métabolique dans l'utilisation de ceux-ci (Radwan, 2003).

Cette étude avait pour objectif d'évaluer l'impact des Aliments Thérapeutiques Prêts à l'Emploi (ATPE) sur les paramètres biochimiques et anthropométriques des enfants malnutris aiguë sévère à l'Institut de Nutrition et Santé de l'Enfant de Guinée (INSE) qui a pour rôle de faire la récupération nutritionnelle des enfants malnutris sévères en utilisant les laits thérapeutiques F75 et F100.

\section{MATERIEL ET METHODES}

Il s'agissait d'une étude prospective descriptive de 6 mois portant sur des enfants souffrant de malnutrition aiguë sévère, pris en charge au Centre de Récupération et d'Education Nutritionnel Intensif (CRENI) de l'institut de Nutrition et de Santé de l'Enfant (INSE) de Guinée du 2 septembre 2013 au 27 février 2014. 
Pour être inclus dans cette étude, les enfants (âgés de 0 à 5 ans) devaient être pris en charge au CRENI pour malnutrition aigüe sévère et bénéficier d'aliments thérapeutiques prêts à l'emploi (ATPE) pour leur prise en charge ; leur recrutement était basé sur le fait d'avoir un rapport poids/taille $\mathrm{P} / \mathrm{T} \leq$ à $3 \mathrm{z}$ Score et ou un périmètre brachial $\mathrm{PB} \leq$ à 115 $\mathrm{mm}$ ainsi que par la présence ou non d'œdèmes bilatéraux aux membres inférieurs.

Pour la prise en charge de ces enfants, les phases ci-après ont été suivies :

La phase I dite de stabilisation visant à résorber les œdèmes, à restaurer les tissus, ainsi qu'à restaurer le bon fonctionnement des organes. Elle requiert de traiter les infections et les autres problèmes médicaux, d'apporter suffisamment de calories et de nutriments afin d'enrayer la fonte musculaire, des graisses et corriger le déséquilibre électrolytique à l'aide du lait thérapeutique F-75, qui contient 75 kilocalories et 0,9 gramme de protéines pour 100 millilitres de lait.

La phase II dite de réhabilitation durant laquelle les enfants reçoivent des apports supplémentaires en calories et en nutriments qui les aident à prendre rapidement $\mathrm{du}$ poids et à rattraper leur retard de croissance à l'aide du lait thérapeutique $\mathrm{F}$ 100 , qui contient 100 kilocalories et 3 grammes de protéines pour 100 millilitres de lait.

Les données sociodémographiques ont été collectées à l'aide d'une fiche d'enquête préétablie lors d'interviews avec les mères de ces enfants, suivie de l'enregistrement de leurs données cliniques, anthropométriques et biologiques par un médecin. En effet, chaque enfant à l'admission a été examiné par un médecin pour son évaluation ; cette évaluation concernait essentiellement la prise de la taille, $\mathrm{du}$ poids et l'appréciation des autres signes cliniques. Tous les enfants qui avaient un rapport $\mathrm{P} / \mathrm{T} \leq$ à $3 \mathrm{z}$ score ou présentant des œdèmes bilatéraux ont été déclarés dans un état de malnutrition aiguë sévère.

\section{Analyse statistique}

Ces données recueillies ont ensuite été saisies et analysées en utilisant le logiciel Statistical Package for Social Science (SPSS) version 21. Des analyses statistiques ont été réalisées à l'aide du test statistique de Kruskall-Wallis (analyse non paramétrique), avec un seuil de significativité de 5\% $(0,05)$.

\section{Considérations éthiques}

Le protocole de cette étude a été approuvé par la Chaire de Biochimie, Nutrition et Toxicologie Clinique du département Pharmacie de la Faculté de Médecine de l'Université Gamal Abdel Nasser de Conakry.

Les parents des enfants qui y ont participé ont bien été informés sur ses objectifs et ont donné leur consentement libre et éclairé avant d'être soumis au questionnaire.

\section{RESULTATS}

$\mathrm{Au}$ total, 41 enfants souffrant de malnutrition aigüe sévère ont été recensés. La majorité d'entre eux (66\%) étaient âgés entre 13 et 36 mois; 18 (44\%) étaient de sexe masculin et $23(56 \%)$ de sexe féminin ; $45 \%$ d'entre eux avaient été sevrés dans les conditions requises (entre 4 et 6 mois) contre $11 \%$ qui l'avaient été hâtivement et $19 \%$ qui l'avaient été tardivement. Tous ces enfants (100\%) avaient été hospitalisés. Parmi eux, 27 (66\%) avaient suivi le traitement contre la malnutrition jusqu'à la fin de la phase 1 et 10 (24\%) jusqu'à la fin de la phase 2 du traitement. $\mathrm{La}$ durée moyenne d'hospitalisation était de $24 \pm 11$ jours (Tableau 1).

S'agissant des caractéristiques sociodémographiques des mères en charge de ces enfants, 38 (93\%) d'entre elles étaient mariées ; 38 (93\%) autres étaient des ménagères contre $3(7 \%)$ qui étaient des vendeuses; 37 (90\%) d'entre elles n'avaient jamais été scolarisées contre 1 seule $(2 \%)$ qui avait étudié jusqu'au secondaire (Tableau 1).

Sur le plan clinique, ces enfants avaient été reçus en consultation majoritairement pour des candidoses digestives (100\%), de la fièvre (93\%), une anorexie (66\%), une bronchite aigüe (66\%), une toux (61\%), des vomissements et des diarrhées chez $56 \%$ d'entre eux. Les œdèmes aux membres inférieurs n'étaient présents que chez $10 \%$ d'entre eux. 
Paramètres biochimiques chez les enfants malnutris avant le début du traitement

Sur le plan biochimique, tous ces enfants (100\%) souffraient de carences d'éléments importants dans le sang avant de débuter le traitement contre la malnutrition : $22 \%$ d'entre eux avaient une hypoalbuminémie; $61 \%$ étaient en hypoglycémie; $63 \%$ avaient une hypo protéinémie totale; $66 \%$ une hypocalcémie ; $80 \%$ une hyponatrémie; $41 \%$ une hypokaliémie; $39 \%$ une hypochlorémie et $78 \%$ une hyposidérémie (Tableau 2).

\section{Paramètres biochimiques chez les enfants malnutris à mi-parcours du traitement}

Au terme de la phase 1 (après la prise du lait thérapeutique F75), 18 (67\%) des 27 enfants qui avaient suivis le traitement jusqu'à ce stade avaient une albuminémie normale; $15(56 \%)$ une glycémie normale et $17(63 \%)$ une natrémie normale. Alors que $13(48 \%)$ avaient hypoprotéinémie totale ; 14 (52\%) une hypocalcémie et 17 (63\%) une hyposidérémie. Cependant la kaliémie était élevée chez 16 (59\%) d'entre eux (Tableau 3).

\section{Paramètres biochimiques chez les enfants malnutris à la sortie}

A l'issue de la phase 2 (après la prise $\mathrm{du}$ lait thérapeutique F100), nous avons constaté une normalisation des paramètres biologiques. Parmi les 10 enfants qui avaient suivi le traitement contre la malnutrition jusqu'à ce stade, $7(70 \%)$ avaient une albuminémie normale; $6(60 \%)$ avaient une glycémie normale, une protéinémie totale normale et une calcémie normale ; 4 (40\%) d'entre eux avait une Sidérémie normale; tandis qu'eux tous avaient une calcémie normale. Cependant, $8(80 \%)$ et $5(50 \%)$ d'entre eux avaient respectivement une hyperkaliémie et une hyperchlorémie (Tableau 4).

Les médianes de l'albuminémie (Figure 1), de la calcémie (Figure 2), de la natrémie (Figure 3), de la chlorémie (Figure 4), de la kaliémie (Figure 5), et de la sidérémie (Figure 6) ont connu une augmentation significative $(p<0,05)$ au cours des différentes phases du traitement. Ce qui montre que la prise en charge de ces enfants par les ATPE avait permis une amélioration sensible de leurs paramètres biochimiques sanguins. L'augmentation du taux médian de la protéine totale, de la glycémie et du calcium n'était pas statistiquement significative ( $>0,05)$ au cours des différentes phases du traitement.

Etat de la récupération des paramètres biochimiques et staturo-pondéraux chez les enfants à la sortie

Du point de vue staturo-pondéral, tous ces enfants $(100 \%)$ avaient un déficit pondéral à leur arrivée avec un rapport poids/taille en dessous de -2 Z-Score. A l'issue du traitement, leurs paramètres staturo-pondéraux s'étaient nettement améliorés et variaient désormais entre 7,0 à 9,6 kg, correspondants à des gains de poids de 800 à $1600 \mathrm{~g}$ soit $90 \%$ et $102 \%$ des poids cibles respectivement (Tableau 4).

Tableau 1: Répartition des enfants et de leurs mères en fonction de leurs caractéristiques sociodémographiques.

\begin{tabular}{lcc}
\hline Caractéristiques & Effectif $(\mathbf{N}=\mathbf{4 1})$ & Pourcentage (\%) \\
\hline Age (mois) & & \\
3-12 & 13 & 32,0 \\
13-36 & 27 & 66,0 \\
$37-59$ & 1 & 2,0 \\
\hline Sexe & & \\
Masculin & 18 & 44,0 \\
Féminin & 23 & 56,0 \\
\hline Type de sevrage (période en mois) & & \\
<4 (hâtif) & 5 & 11,0 \\
4-6 (normal) & 18 & 45,0 \\
>6 (tardif) & 12 & 19,0
\end{tabular}




\begin{tabular}{lcc} 
Inconnue & 6 & 15,0 \\
\hline Situation matrimoniale de la mère & 2 & \\
Célibataire & 38 & 5,0 \\
Mariée & 1 & 93,0 \\
Divorcée & 3 & 2,0 \\
\hline Profession de la mère & 38 & 7,0 \\
Vendeuse & & 93,0 \\
Ménagère & 37 & 90,0 \\
\hline Niveau d'étude de la mère & 3 & 7,0 \\
Sans niveau & 1 & 2,0 \\
Primaire & &
\end{tabular}

Tableau 2: Etat des paramètres biochimiques des enfants malnutris avant le début du traitement de la malnutrition.

\begin{tabular}{lccccccc}
\hline \multirow{2}{*}{$\begin{array}{l}\text { Paramètres } \\
\text { biochimiques }\end{array}$} & \multicolumn{9}{c}{ Valeurs } & \multirow{2}{*}{ Total } \\
\cline { 2 - 6 } & \multicolumn{2}{c}{ Basses } & \multicolumn{2}{c}{ Normales } & \\
\cline { 2 - 6 } & nombre & \% & nombre & \% & nombre & \% & \\
\hline Albumine & 9 & 22,0 & 22 & 54,0 & 10 & 24,0 & 41 \\
Glycémie & 25 & 61,0 & 13 & 32,0 & 3 & 7,0 & 41 \\
Protéine totale & 26 & 63,0 & 9 & 22,0 & 6 & 15,0 & 41 \\
Calcium & 27 & 66,0 & 11 & 27,0 & 3 & 7,0 & 41 \\
Sodium & 33 & 80,0 & 7 & 17,0 & 1 & 2,0 & 41 \\
Potassium & 17 & 41,0 & 18 & 44,0 & 6 & 15,0 & 41 \\
Chlore & 16 & 39,0 & 18 & 44,0 & 7 & 17,0 & 41 \\
Fer sérique & 32 & 78,0 & 8 & 19,0 & 1 & 2,0 & 41 \\
\hline
\end{tabular}

Tableau 3: Etat des paramètres biochimiques des enfants malnutris à mi-parcours du traitement de la malnutrition.

\begin{tabular}{lccccccc}
\hline \multirow{2}{*}{$\begin{array}{l}\text { Paramètres } \\
\text { Biochimiques }\end{array}$} & \multicolumn{9}{c}{ Valeurs } & Total \\
\cline { 2 - 6 } & \multicolumn{2}{c}{ Basses } & \multicolumn{2}{c}{ Normales } & \multicolumn{2}{c}{ Elevées } & \\
\cline { 2 - 6 } & nombre & $\%$ & nombre & $\%$ & nombre & $\%$ & \\
\hline Albumine & 4 & 15,0 & 18 & 67,0 & 5 & 18,0 & 27 \\
Glycémie & 11 & 41,0 & 15 & 56,0 & 1 & 4,0 & 27 \\
Protéine totale & 13 & 48,0 & 11 & 41,0 & 3 & 11,0 & 27 \\
Calcium & 14 & 52,0 & 13 & 48,0 & 0 & 0,0 & 27 \\
Sodium & 10 & 37,0 & 17 & 63,0 & 0 & 0,0 & 27 \\
Potassium & 0 & 0,0 & 11 & 41,0 & 16 & 59,0 & 27 \\
Chlore & 3 & 11,0 & 13 & 48,0 & 11 & 41,0 & 27 \\
Fer sérique & 17 & 63,0 & 7 & 26,0 & 3 & 11,0 & 27 \\
\hline
\end{tabular}


Tableau 4: Etat des paramètres biochimiques des enfants malnutris à l'issue du traitement de la malnutrition.

\begin{tabular}{lccccccc}
\hline \multirow{2}{*}{$\begin{array}{l}\text { Paramètres } \\
\text { biochimiques }\end{array}$} & \multicolumn{6}{c}{ Basses } & \multicolumn{2}{c}{ Normaleurs } & Total \\
\cline { 2 - 6 } & nombre & \% & nombre & \% & Nombre & \% & \\
\hline Albumine & 1 & 10,0 & 7 & 70,0 & 2 & 20,0 & 10 \\
Glycémie & 4 & 40,0 & 6 & 60,0 & 0 & 0,0 & 10 \\
Protéine totale & 2 & 20,0 & 6 & 60,0 & 2 & 20,0 & 10 \\
Calcium & 2 & 20,0 & 6 & 60,0 & 2 & 20,0 & 10 \\
Sodium & 0 & 0,0 & 10 & 100,0 & 0 & 0,0 & 10 \\
Potassium & 0 & 0,0 & 2 & 20,0 & 8 & 80,0 & 10 \\
Chlore & 1 & 10,0 & 4 & 40,0 & 5 & 50,0 & 10 \\
Fer sérique & 2 & 20,0 & 4 & 40,0 & 4 & 40,0 & 10 \\
Transferrine & 1 & 10,0 & 9 & 90,0 & 0 & 0,0 & 10 \\
\hline
\end{tabular}

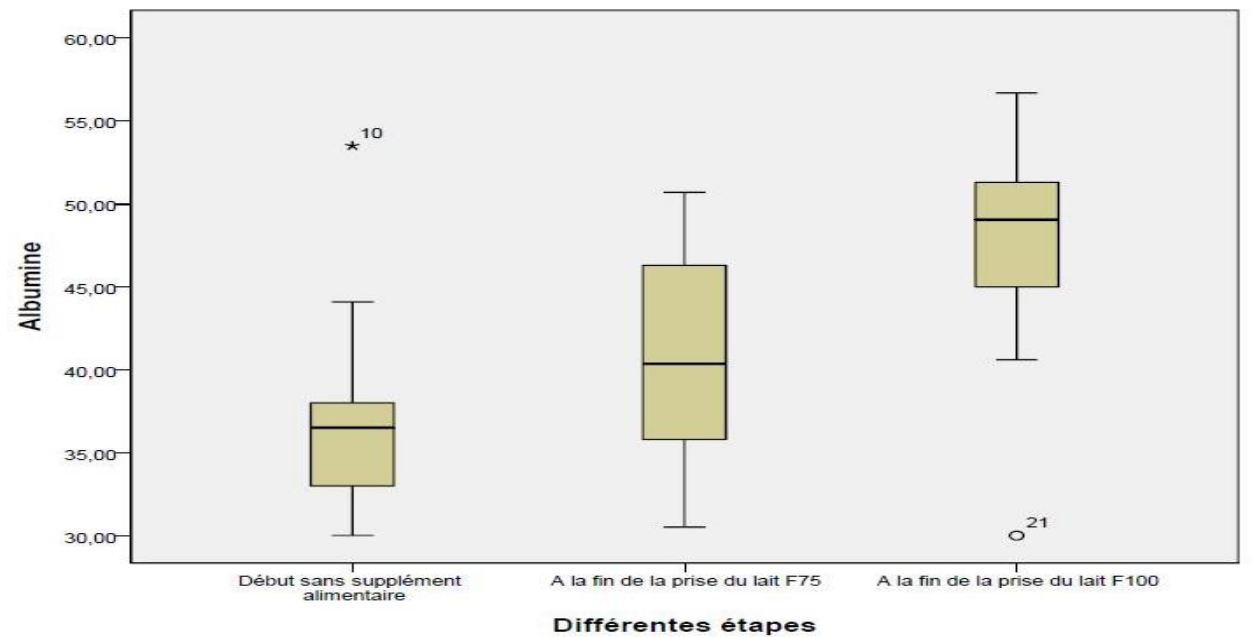

Figure 1: Variation des médianes de l'albuminémie au cours des différentes phases de récupération.

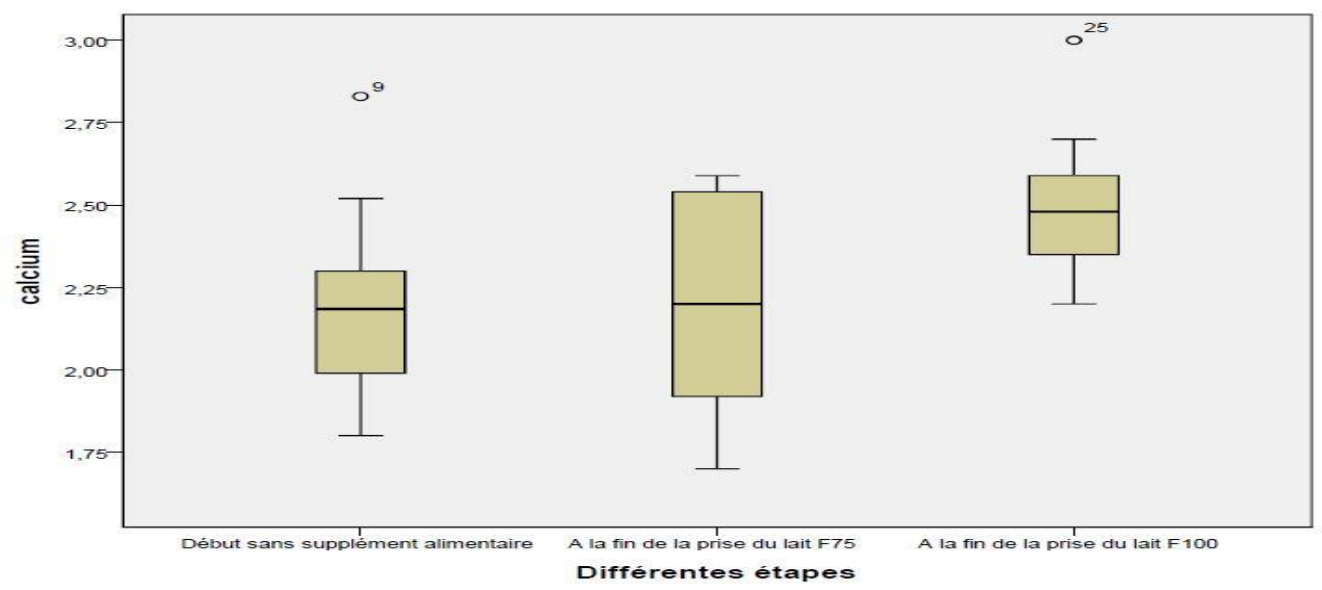

Figure 2: Variation des médianes de la calcémie au cours des différentes phases de récupération. 


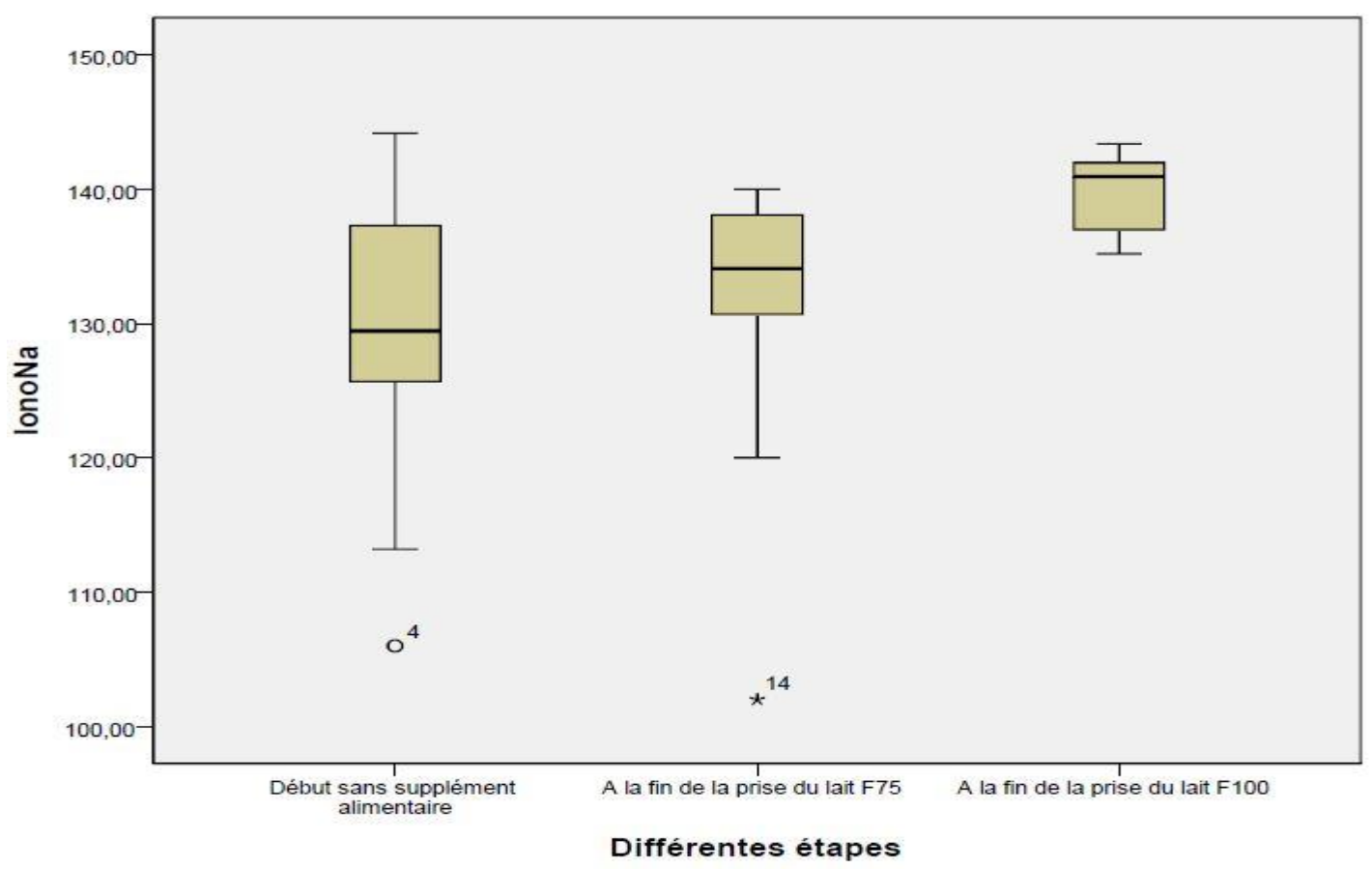

Figure 3: Variation des médianes de la natrémie au cours des différentes phases de récupération.

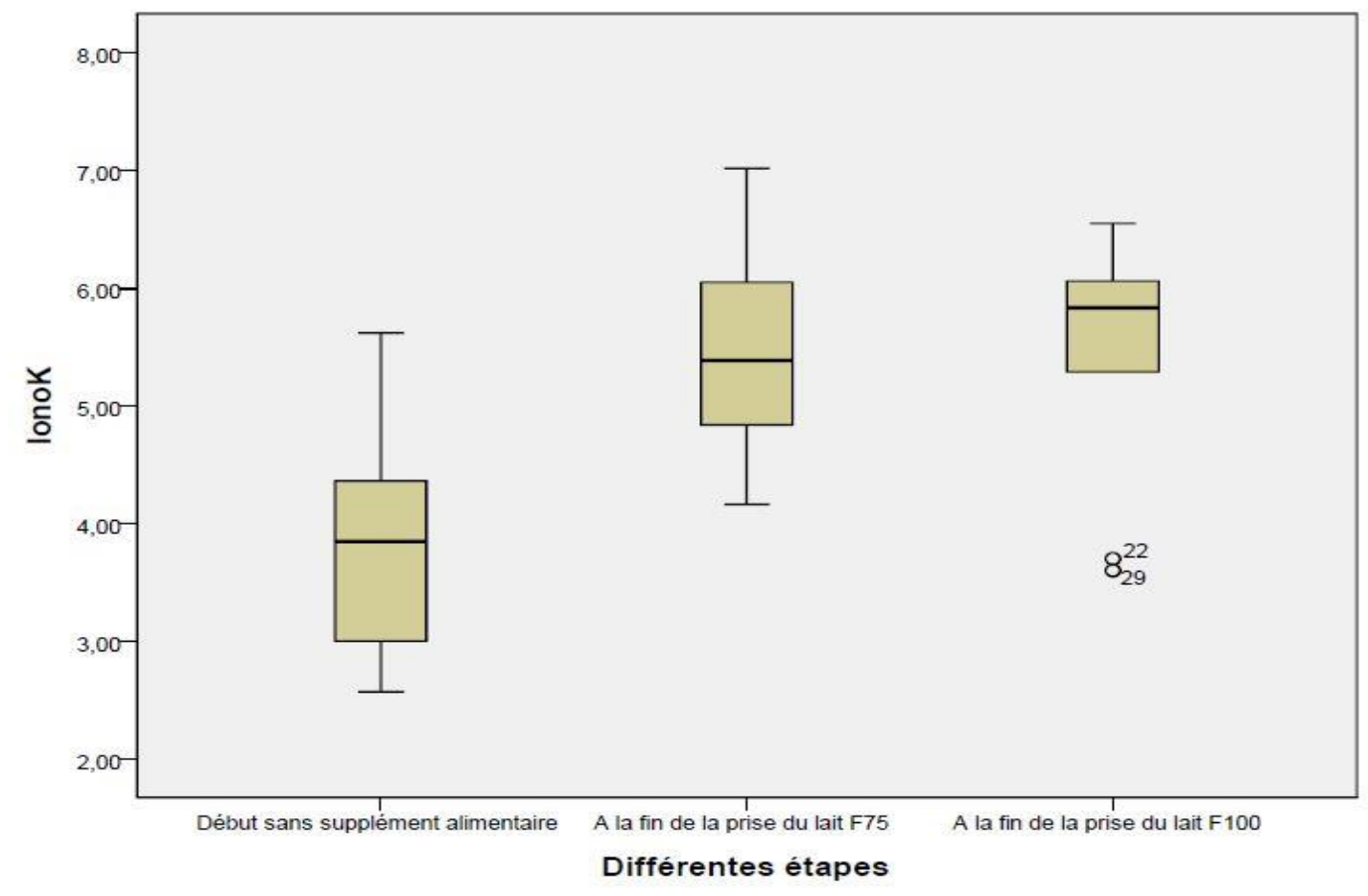

Figure 4: Variation des médianes de la kaliémie au cours des différentes phases de récupération. 


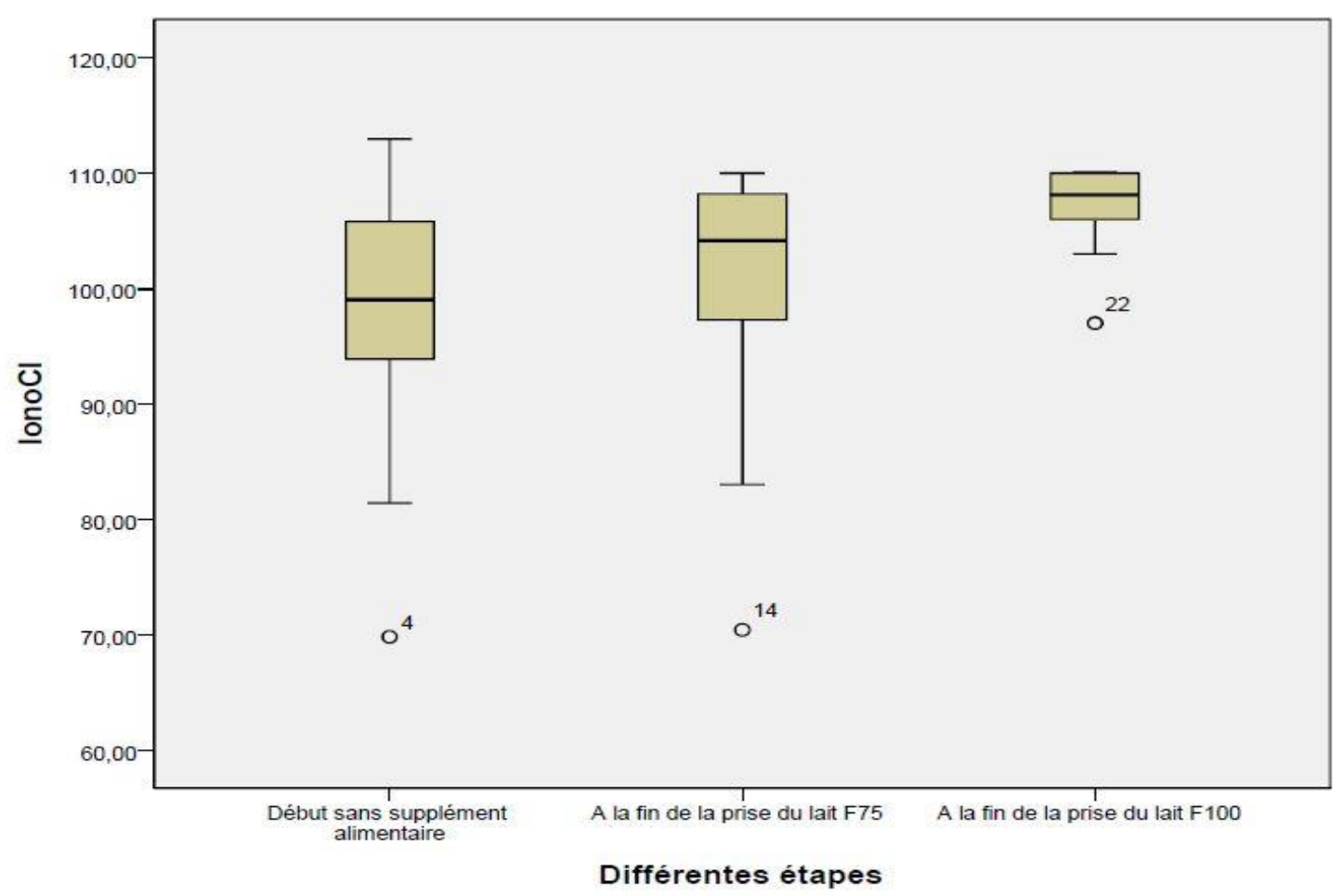

Figure 5 : Variation des médianes de la chlorémie au cours des différentes phases de récupération.

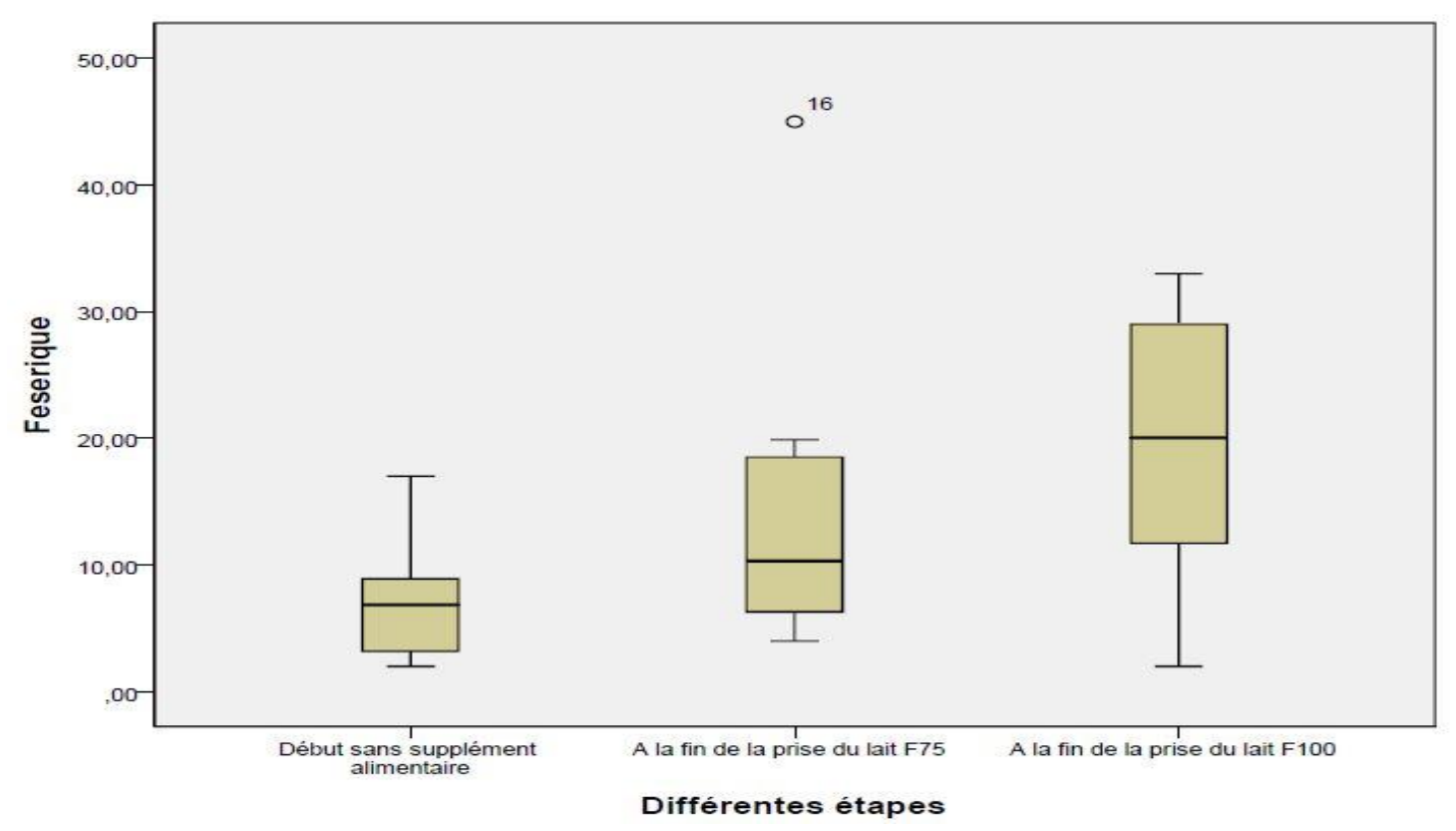

Figure 6 : Variation des médianes de la sidérémie au cours des différentes phases de récupération. 
Tableau 5 : Gain pondéral chez les enfants récupérés à la sortie.

\begin{tabular}{ccccccc}
\hline Sexes & $\begin{array}{c}\text { P/T z score } \\
\text { rentrée }\end{array}$ & $\begin{array}{c}\text { P/A kg/mois } \\
\text { Rentrée }\end{array}$ & Poids sortie kg & Poids cible kg & Gain pondéral G & \% pondéral \\
\hline F & $<-3$ & 0,45 & 7,0 & 7,1 & 800 & 90 \\
F & $<-4$ & 0,23 & 8,3 & 9,0 & 1400 & 92 \\
F & $<-4$ & 0,28 & 6,8 & 6,9 & 1100 & 99 \\
M & $<-4$ & 0,54 & 8,4 & 8,4 & 1900 & 100 \\
M & $<-2$ & 0,42 & 8,3 & 8,2 & 700 & 101 \\
F & $<-3$ & 0,33 & 7,6 & 7,8 & 1000 & 97 \\
F & $<-4$ & 0,48 & 7,6 & 7,5 & 1800 & 101 \\
F & $<-3$ & 0,40 & 8,3 & 8,2 & 1100 & 101 \\
M & $<-4$ & 0,26 & 8,0 & 8,1 & 1600 & 98 \\
M & $<-3$ & 0,33 & 9,6 & 9,4 & 1600 & 102 \\
\hline
\end{tabular}

\section{DISCUSSION}

Dans notre échantillon, les enfants malnutris étaient majoritairement de sexe féminin (56\%) contre $44 \%$ de sexe masculin. Ces résultats sont proches de ceux rapportés par Ousmane Ouédraogo (2012) au Burkina Faso qui avait rapporté $51,7 \%$ de filles contre $48,3 \%$ de garçons dans son étude sur un échantillon de 60 enfants concernant la prise en charge des enfants malnutris au Centre d'Education et de Récupération Nutritionnelle (CREN) de Ouagadougou (Ouédraogo, 2012).

La tranche d'âge la plus touchée par la malnutrition était celle des 13 - 36 mois avec $66 \%$ des cas ; Sall et al. (2000) lors d'une étude sur la récupération nutritionnelle chez 2917 enfants malnutris au Sénégal, avaient observé que $63 \%$ de ces enfants étaient de la tranche d'âge des 12-24 mois (Sall et al., 2000). Cela pourrait se justifier par le fait qu'à cet âge, l'alimentation de ces enfants ne soit plus contrôlée attentivement par leurs parents tandis que leurs besoins nutritionnels sont alors plus importants que jamais.

Les symptômes qui avaient emmené les mères en charge de ces enfants à consulter étaient majoritairement les candidoses digestives (100\%), la fièvre (93\%), l'anorexie $(90 \%)$, la bronchite aigüe $(66 \%)$, la toux (61\%), les vomissements et les diarrhées $(56 \%)$; alors que les œed̀mes aux membres inférieurs n'étaient présents que chez $10 \%$ d'entre eux. Ces symptômes dont souffraient ces enfants sont consécutifs à la malnutrition protéino-calorique, pouvant être dus à un mauvais sevrage ou à l'inattention de leurs parents vis-à-vis de leur état nutritionnel. Nos résultats similaires à ceux rapportés par Diop et al. (2004) à Dakar (Sénégal) lors d'une étude menée sur l'efficacité du transfert de récupération nutritionnelle à domicile des enfants sévèrement malnutris où, $60 \%$ des enfants présentaient une diarrhée, des vomissements, de la fièvre ainsi que des infections respiratoires aiguës à l'admission au Centre de récupération nutritionnelle (Diop et al., 2004).

Dans notre étude, les paramètres staturo-pondéraux de tous les 41 enfants (100\%) qui avaient un déficit pondéral à leur arrivée avec un rapport poids/taille en dessous de -2 Z-Score ont connu une nette amélioration après traitement avec un gain pondéral de 800 à $1600 \mathrm{~g}$. En Ethiopie, Yebyo et al. (2013) ont rapporté les résultats similaires dans leur étude sur le traitement ambulatoire de 628 enfants malnutris sévères, qui a trouvé une augmentation de $21,4 \%$ de poids des enfants après les phases d'intervention $\mathrm{du}$ traitement à base des aliments thérapeutiques (Yebyo et al., 2013).

Cette bonne croissance staturopondérale observée chez les enfants à la sortie correspond-elle à une bonne amélioration des 
paramètres biochimiques? En effet, si les paramètres staturo-pondéraux des enfants malnutris pris en charge par les ATPE sont dans les normes à la sortie, les paramètres biochimiques montrent tout autre visage. Au terme de la phase1 et de la phase 2 du traitement avec les laits thérapeutiques F75 et F100, nous avons constaté une normalisation progressive de l'albuminémie avec des pourcentages atteignant 67 et $70 \%$ respectivement.

$\mathrm{Au}$ cours des différentes phases du traitement avec F75 et F100, nous avons constaté une augmentation significative du taux médian de certains paramètres biochimiques notamment de l'albuminémie de 42,3 g/l, du taux médian de sodium de $136,50 \mathrm{mmol} / \mathrm{l}$, du taux médian du potassium de 4,92 mmol/l, du taux médian du chlore de $105,90 \mathrm{mmol} / \mathrm{l}$ et du taux médian de fer sérique de 10,55 $\mu \mathrm{mol} / \mathrm{l}$. Cependant, cette augmentation de la médiane des paramètres reste non significative pour la glycémie, du Calcium et de la transferrine chez les enfants après les différentes phases de la récupération. Au Sénégal, Diop et al. (2004) ont rapporté une augmentation significative du taux d'hémoglobine et d'hématocrite chez les enfants sous F100 à la fin de la récupération. A l'inverse, chez les enfants malnutris sous RTUF (Ready-to-Use Therapeutic Food), les taux d'hémoglobine et d'hématocrite n'ont pas connu de changement significatif au cours de la récupération (Diop et al., 2004).

Sur le plan biochimique, nous avons constaté que $22(54 \%)$ des enfants malnutris avaient une normo-albuminémie à leur arrivée et $46 \%$ avaient une perturbation du foyer albuminique. Cette perturbation du foyer albuminique présentait une disparité variable en ce sens que $22 \%$ des enfants avaient une hypo-albuminémie tandis que $24 \%$ d'entre eux avaient une hyper-albuminémie. Cependant, la protéinémie ne suivait pas la tendance albuminique, puisque $63 \%$ de ces enfants malnutris avaient une protéinémie totale basse contre seulement $22 \%$ et $15 \%$ qui avaient une protéinémie totale normale et élevée respectivement; ce qui représente, globalement, une perturbation de foyer protéinique de $78 \%$. La dynamique liée au traitement par les aliments thérapeutiques montre également une tendance positive de la protéinémie, car elle est passée de $22 \%$ d'enfants normo-protéiques à l'arrivée à $60 \%$ à la sortie. Cela se traduit sur le plan biologique par une variation globalement positive de la protéinémie de $63,33 \pm 12,53$ $\mathrm{g} / \mathrm{l}$ à 70,52 $\pm 10,11 \mathrm{~g} / 1$ en moyenne.

Dans notre étude, $66 \%, 80 \%, 41 \%$ et $39 \%$ des enfants malnutris de notre échantillon à la rentrée avaient une des valeurs basses en calcémie, en une natrémie, une en kaliémie et en une chlorémie basse respectivement.

L'une des limites de notre étude est liée á la taille de l'échantillon qui a été difficile à obtenir à cause des difficultés liées aux parents comme des contraintes financières, l'éloignement, mauvaise perception de la prise en charge des enfants malnutris et les perdus de vue des enfants malnutris pendant la période d'étude.

\section{Conclusion}

Globalement, nous avons constaté que les enfants avaient tous récupéré d'un point de vue staturo-pondéral mais des disparités ont été observées au niveau de leurs paramètres biochimiques qui variaient d'un enfant à l'autre. En effet, sur les 10 enfants qui avaient suivi les trois étapes du traitement contre la malnutrition jusqu'au bout, seuls 3 présentaient un statut biochimique normal à la sortie. Cet état de fait doit attirer l'attention des agents chargés de la prise en charge nutritionnelle de ces enfants malnutris au CRENI de l'INSE de Donka, pour veiller à la normalisation de leurs paramètres biochimiques, notamment leur kaliémie et de leur chlorémie à travers un suivi en ambulatoire après leur sortie. La promotion de l'utilisation des aliments thérapeutiques prêts à l'emploi (ATPE) dans la prise en charge des malnutritions aiguës sévères devrait donc être encouragée en veillant toutefois à normaliser les paramètres ioniques après coup dont les perturbations peuvent entraîner des complications fâcheuses. L'utilisation des produits locaux pour leur fabrication serait 
aussi judicieuse, quand on sait que la République de Guinée en regorge de ressources suffisantes pour le faire.

\section{CONFLITS D'INTERETS}

Cette étude ne fait l'objet d'aucun conflit d'intérêts. Ses données sont authentiques et n'ont jamais été publiées dans une revue scientifique auparavant.

\section{CONTRIBUTIONS DES AUTEURS}

FT a participé à la conception de l'étude, à sa mise en œuvre, l'interprétation des données et la rédaction de l'article ; MSB et MMS ont participé à la conception de l'étude, à sa mise en œuvre et la rédaction de l'article, SS a analysé et interprété les résultats, rédaction $\mathrm{BL}$ et $\mathrm{ASD}$ ont coordonné l'étude, participé à la correction et la relecture du manuscrit. ATS a participé à la mise en œuvre et la rédaction de l'article.

\section{REFERENCES}

Ashworth A, Khanum S, Jackson A, Schofield C. 2003. Guidelines for the Inpatient Treatment of Severely Malnourished Children; World Health Organization: Geneva, Switzerland.

Diop El, Dossou NI, Briend A, Yaya MA, Ndour MM, Wade S. 2004. Home-based rehabilitation for severely malnourished children using locally made ready-to-use therapeutic food (R11JF). Pmoetxfings of the Wood Congress of Gastroenterology, Hepatology and Nutrition, Paris, France.

Diop El HI, Dossou NI, Ndour MM, Briend A, Wade S. 2003. Comparison of the efficacy of a solid ready-to-use food and a liquid, milk-based diet for the rehabilitation of severely malnourished children: a randomized trial. Am J Clin Nutr., 78(2): 302-307.

Fergusson P, Tomkins A. 2009. HIV prevalence and mortality among children undergoing treatment for severe acute malnutrition in sub-Saharan Africa: a systematic review and meta-analysis. Transactions of the Royal Society of Tropical Medicine and Hygiene, 103(6): 541-548.

Ministère de la Santé et de L'Hygiène Publique de Guinée (MSHP). 2008. Prise en charge de la malnutrition aigüe, politique nationale d'alimentation, Commission UE/UNICEF 2008.

OMS et UNICEF. 2009. Normes de croissance OMS et identification de la malnutrition aiguë sévère chez l'enfant. Déclaration commune de l'OMS et de l'UNICEF.

Ouédraogo O. 2013. Prise en charge des enfants malnutris admis au CREN du centre medical saint camille de Ouagadougou (Burkina Faso) : maitrise en nutrition humaine et technologie alimentaire Université de Ouagadougou. 2012. Mémoire Online 2000-2013.

Radwan F. 2003. Les marqueurs biochimiques de la nutrition, Nutrition, Science en Evolution, 1: 15.

Sall MG, Badji ML, Martin SL. 2000. Récupération nutritionnelle en milieu hospitalier régional : Le cas de l'hôpital régional de Kaolack (Sénégal). Médecine d'Afrique Noire A. 47(12): 525-527.

World Health Organization (WHO). 2013. Guideline: Updates on the Management of Severe Acute Malnutrition in Infants and Children. World Health Organization: Geneva, Switzerland.

Yebyo HG, Kendall C, Nigusse D, Lemma W. 2013. Outpatient therapeutic feeding program outcomes and determinants in treatment of severe acute malnutrition in Tigray, northern Ethiopia: A retrospective cohort study. PLOS ONE. 8: $251 . \quad$ DOI: 10.1371/journal.pone.0065840. 